\title{
TOMOGRAPHIC APPROACH FOR PARAMETRIC ESTIMATION OF LOCAL DIFFUSIVE SOURCES AND APPLICATION TO HEAT DIFFUSION
}

\author{
Ivana Jovanović ${ }^{\dagger}$, Luciano Sbaiz $^{\dagger}$ and Martin Vetterli ${ }^{\dagger \S}$ \\ † School of Computer and Communication Sciences \\ Ecole Polytechnique Fédérale de Lausanne (EPFL), CH-1015 Lausanne, Switzerland \\ $\S$ Department of Electrical Engineering and Computer Sciences \\ University of California at Berkeley (UCB), Berkeley, CA 94720, USA \\ email: \{ivana.jovanovic, luciano.sbaiz, martin.vetterli\}@epfl.ch
}

\begin{abstract}
We consider localized instantaneous sources that reside in a 2D diffusive environment. Our goal is to reconstruct the induced field from the measurements obtained by distributed sensors. Although the field is non-bandlimited, we capitalize on the fact that it is completely determined by a finite number of parameters to develop a method that allows perfect reconstruction. We demonstrate how these results can be applied in practice in the particular case of heat diffusion. Simulation results confirm the effectiveness of the method.
\end{abstract}

Index Terms - Tomography, FRI, heat, diffusion, Radon

\section{INTRODUCTION}

Sensor networks are typically used to monitor a physical quantity over space and time. A particular challenge of the sampling problem in sensor networks arises from the fact that the considered signals are usually neither bandlimited in space nor in time. While the filtering over time can be locally performed at each sensor, filtering over space remains a serious sampling limitation. Still, depending on the physical properties of the environment, we can identify the interactions between the sources and the environment as a specific filtering operation. Examples are found in diffusion processes, where the initially localized release of a point source is spread (filtered) over space in a Gaussian manner. Practical situations include heat diffusion, gas diffusion, pollutants diffusion in water, etc. Incorporating the physical characteristics of the field in the sampling scheme will help us to go beyond the standard sampling results.

In this work, we consider localized instantaneous sources that reside in a 2D diffusive environment. Due to their localization in space and time, the sources can be modeled as a set of weighted Diracs. After the activation of a source, the induced field, although non-bandlimited, is completely determined by a finite set of parameters, namely, the source location, its time origin and the total amount released. Recent results in [1] extend the original Shannon theory to the sampling of signals characterized by a finite number of parameters, so called the signals with finite rate of innovation (FRI). The direct reconstruction approach is exchanged for the reconstruction of the finite number of parameters. Intuitively, only a finite number of samples is required for perfect reconstruction. The exact sampling

This research was supported by the National Competence Center in Research on Mobile Information and Communication Systems (NCCR-MICS, http://www.mics.org), a center supported by the Swiss National Science Foundation under grant number 5005-67322. schemes, for large classes of the 1-D and 2-D FRI signals, have been developed in $[1,2,3]$.

In this work, we will extend some of the results formulated in [4] for a set of 2-D Diracs. The property of the Radon transform projections is exploited in order to reduce the 2-D problem to its one-dimensional equivalent. Two general scenarios, when the Diracs appear at the same but unknown time and when they appear at different times, are envisioned. The algorithms can be extended to track the appearance of the new sources, as well.

While these results are applicable to any diffusive environment, obtaining the Radon transform projections still remains the practical challenge. However, we demonstrate that in the case of heat diffusion the corresponding projections can be estimated from acoustic tomography measurements. Time of flight measurements are used for this purpose. Possible applications range from large scale environmental monitoring to small scale heat control in medical therapy. Our simulation results confirm the applicability of the method.

\section{PROBLEM STATEMENT}

We consider the problem of reconstructing a 2-D field induced by sources localized in space and time. Moreover, we want to compute the positions of the sources, the origin of time and the total amount released. A set of 3-D weighted Diracs is used to model the sources:

$$
s(x, y, t)=\sum_{k=0}^{K-1} c_{k} \delta\left(x-x_{k}, y-y_{k}, t-t_{k}\right) .
$$

All diffusive processes are governed by diffusion, or equivalently the heat equation. From this equation, we know that a concentrated deposit diffuses away in a Gaussian manner as described by the 2-D Green's function, also known as the 2-D heat kernel:

$$
h(x, y, t)=\frac{1}{4 \pi D t} e^{-\frac{x^{2}+y^{2}}{4 D t}},
$$

where $D$ is a diffusion constant. At any point we can compute the field as a convolution of the local sources with the heat kernel:

$g(x, y, t)=[s * h](x, y, t)=\sum_{k=0}^{K-1} \frac{c_{k}}{4 \pi D\left(t-t_{k}\right)} e^{-\frac{\left(x-x_{k}\right)^{2}+\left(y-y_{k}\right)^{2}}{4 D\left(t-t_{k}\right)}}$.

The resulting field is simply a weighted sum of $K$ 2-D Gaussians that diffuse over time. Notice also that $g(x, y, t)$ is completely determined by the parameters of the sources $\left\{c_{k}, x_{k}, y_{k}\right\}$ and the time origins $t_{k}$. 


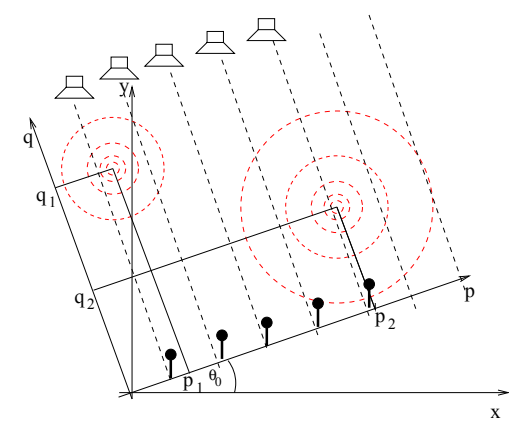

Fig. 1. Sampling of 2-D Gaussians using the Radon projections. For the perfect reconstruction of 2 Gaussians we need only $2 \cdot 4+1$ samples of the Radon transform.

\section{SOLUTION BASED ON PROJECTIONS}

In this section, we envision three possible scenarios. The first one assumes that all Diracs appear at the same but unknown time. This assumption allows to obtain the exact reconstruction of the field $g(x, y, t)$. In the second scenario, the Diracs appear at different times and only an approximate solution is proposed. In the third scenario we also consider different tracking problems.

\subsection{All sources activated at the same time}

In the following, for the sake of simplicity, we are first going to show the method for estimating the parameters $\left\{c_{k}, x_{k}, y_{k}\right\}$ assuming that the time origin $t_{0}$ is known. The algorithm for estimating $t_{0}$ will follow. The second algorithm does not require the knowledge of $\left\{c_{k}, x_{k}, y_{k}\right\}$ and, in practice, it should be done first.

\subsubsection{Retrieving the parameters $c_{k}, x_{k}$ and $y_{k}$}

Let us assume that all Diracs appear at the same time $t_{0}=0$, and that we observe the system at time $t>0$. This implies that, at the time instant $t$, the variance of the Gaussians is $\sigma^{2}=2 D t$,

$$
g(x, y, t)=\sum_{k=0}^{K-1} \frac{c_{k}}{4 \pi D t} e^{-\frac{\left(x-x_{k}\right)^{2}+\left(y-y_{k}\right)^{2}}{4 D t}} .
$$

Considering the Radon transform of $g(x, y, t)$, defined as:

$$
R g(p, \theta, t)=\int_{-\infty}^{\infty} \int_{-\infty}^{\infty} g(x, y, t) \delta(p-x \cos \theta-y \sin \theta) d x d y
$$

we want to show that a finite number of line integrals in (4) is sufficient for the perfect reconstruction of $g(x, y, t)$.

For a fixed angle $\theta_{0}$ the integral in (4) is computed over the line $\Gamma_{p, \theta_{0}}$ defined by $p=x \cos \left(\theta_{0}\right)+y \sin \left(\theta_{0}\right)$. Let us introduce the $p q$-axes, as shown in Fig. 1, and simplify the computation of the integral. Readily, we have that for any given angle $\theta_{0}$ :

$$
\begin{aligned}
R g\left(p, \theta_{0}, t\right) & =\int_{-\infty}^{\infty} \sum_{k=0}^{K-1} \frac{c_{k}}{4 \pi D t} e^{-\frac{\left(p-p_{k}\right)^{2}+\left(q-q_{k}\right)^{2}}{4 D t}} d q \\
& =\sum_{k=0}^{K-1} \frac{c_{k}}{\sqrt{4 \pi D t}} e^{-\frac{\left(p-p_{k}\right)^{2}}{4 D t}} .
\end{aligned}
$$

The Radon transform $R g\left(p, \theta_{0}, t\right)$ is a weighted sum of $K 1$-D Gaussians. The problem of reconstructing the parameters $c_{k}$ and $p_{k}$ from a finite number of samples of $R g\left(p, \theta_{0}, t\right)$, taken at the positions $p=n L$, for $n=0, \ldots, N-1$ and $N \geq 2 K+1$, is already solved in [1]. For a fixed time $t$, the samples can be written as:

$$
R g[n]=\sum_{k=0}^{K-1}\left(\frac{c_{k}}{\sqrt{4 \pi D t}} e^{-\frac{p_{k}^{2}}{4 D t}}\right) \cdot e^{\frac{n p_{k} L}{2 D t}} e^{-\frac{n^{2} L^{2}}{4 D t}}
$$

If we let $S[n]=R g[n] \cdot e^{\frac{n^{2} L^{2}}{4 D t}}, a_{k}=\frac{c_{k}}{\sqrt{4 \pi D t}} e^{-\frac{p_{k}^{2}}{4 D t}}$ and $u_{k}=$ $e^{\frac{p_{k} L}{2 D t}}$, then (6) is equivalent to

$$
S[n]=\sum_{k=0}^{K-1} a_{k} u_{k}^{n}, \quad n=0, \ldots, N-1 .
$$

Note that, $S[n]$ is a linear combination of the real exponentials $u_{k}$. To compute $u_{k}$ we need to find the so called annihilating filter, that satisfies

$$
\left[\begin{array}{cccc}
S[K] & S[K-1] & \ldots & S[0] \\
S[K+1] & S[K] & \ldots & S[1] \\
\vdots & \vdots & \ddots & \vdots \\
S[2 K] & S[2 K-1] & \ldots & S[K]
\end{array}\right] \cdot\left(\begin{array}{c}
A[0] \\
A[1] \\
\vdots \\
A[K]
\end{array}\right)=\mathbf{0} .
$$

or, shortly

$$
\mathbf{S} \cdot \mathbf{A}=\mathbf{0} .
$$

The method is described in [1]. The roots of the annihilating filter polynomial are exactly the $u_{k}$. The coefficients $a_{k}$ are then directly obtained using (7). From $u_{k}$ we find the positions $p_{k}$ and from $a_{k}$ we find the weights $c_{k}$.

Since the set of locations $p_{k}$ does not itself define the positions of the 2-D Diracs we need one more projection $R g(p, \theta, t)$, e.g. for $\theta=\theta_{0}+\frac{\pi}{2}$. Now, the integration is done along the lines parallel to the $p$-axis:

$$
R g\left(q, \theta_{0}+\pi / 2, t\right)=\sum_{k=0}^{K-1} \frac{c_{k}}{\sqrt{4 \pi D t}} e^{-\frac{\left(q-q_{k}\right)^{2}}{4 D t}} .
$$

Sampling the signal $R g\left(q, \theta_{0}+\pi / 2, t\right)$ at $q=n L$, for $n=0, \ldots, N-$ 1 and $N \geq 2 K+1$, and applying the annihilating filter method, we can first compute $q_{k}$ and then the weights $c_{k}$. By comparing the weights computed from the first and second projection one we will be able for every $p_{k}$ in the first set to associate the corresponding $q_{k}$ in the second set. The proposed method gives the exact positions and weights if all $p_{k}, q_{k}$ and $c_{k}$ are distinct. The case where some of the parameters are equal can be resolved by computing the Radon transform for $K+1$ different angle of $\theta$ [4]. For the case of distinct parameters $c_{k}, p_{k}$ and $q_{k}$, the signal is completely characterized from $2 K+1$ samples of $R g\left(p, \theta_{0}, t\right)$ and $2 K+1$ samples of $R g\left(q, \theta_{0}+\pi / 2, t\right)$.

\subsubsection{Retrieving the time origin}

Assume now that we do not know the time origin $t_{0}$. Let us again consider the annihilating filter formula (8). The entries of the matrix are annihilated by the $K$ roots of the filter $\mathbf{A}=[A[0] \quad \ldots A[K]]^{T}$. The matrix $\mathbf{S}$ has the rank $K$ and it is rank deficient. If we write $S[n]=R g[n] \cdot e^{\frac{n^{2} L^{2}}{4 D t}}=R g[n] \cdot \alpha^{n^{2}}$, then we need to find such $\alpha$ 
for which

$$
\mathbf{S}=\left[\begin{array}{cccc}
R g[K] \alpha^{K^{2}} & R g[K-1] \alpha^{(K-1)^{2}} & \ldots & R g[0] \alpha^{0} \\
R g[K+1] \alpha^{(K+1)^{2}} & R g[K] \alpha^{K^{2}} & \ldots & R g[1] \alpha^{1} \\
\vdots & \vdots & \ddots & \vdots \\
R g[2 K] \alpha^{(2 K)^{2}} & R g[2 K-1] \alpha^{(2 K-1)^{2}} & \ldots & R g[K] \alpha^{K^{2}}
\end{array}\right]
$$

is rank deficient. This is not an easy task especially for noisy data. The straight forward method would be to search for $\alpha$ that minimizes the smallest eigenvalue. This method proves to be very unstable. Instead, we search for $\alpha$ that minimizes the Effective Rank [5]. This new measure represents how the energy is distributed among the directions of the eigenvectors and it can be seen as a continuous extension of the rank. The method largely outperforms the minimum eigenvalue approach (see Sec. 4.2).

\subsection{General case}

Assume that all Diracs appears at different time instants $t_{k}$. Let us rewrite the equation (5) as,

$$
R g\left(p, \theta_{0}, t\right)=\sum_{k=0}^{K-1} c_{k} h\left(p-p_{k}, t-t_{k}\right) u(t)
$$

where $h(p, t)=\frac{1}{\sqrt{4 \pi D t}} e^{-\frac{p^{2}}{4 D t}}$ is a 1-D Gaussian and $u(t)$ is a step function that ensures positive $t$. Taking the Fourier transform, the shifts in space and time translate into the phase shifts:

$$
F\left(\Omega_{p}, \Omega_{t}\right)=\sum_{k=0}^{K-1} c_{k} H\left(\Omega_{p}, \Omega_{t}\right) e^{-j \Omega_{p} p_{k}} e^{-j \Omega_{t} t_{k}} .
$$

The term $H\left(\Omega_{p}, \Omega_{t}\right)$ does not depend on $k$ and can be taken out of the sum. It also represents the 2-D Fourier transform of $h(p, t)$ and can be easily computed. Finally, we get

$$
F\left(\Omega_{p}, \Omega_{t}\right)\left(D \Omega_{p}^{2}+j \Omega_{t}\right)=\sum_{k=0}^{K-1} c_{k} e^{-j \Omega_{p} p_{k}} e^{-j \Omega_{t} t_{k}}
$$

Approximating the Fourier transform $F\left(\Omega_{p}, \Omega_{t}\right)$ with the Discrete Fourier Transform (DFT) coefficients taken at $\Omega_{p}=2 \pi n / N P_{s}$ and $\Omega_{t}=2 \pi m / M T_{s}$, for $n=0, \ldots, N-1, m=0, \ldots, M-1$ where $P_{s}$ and $T_{s}$ are the sampling intervals in space and time respectively, we obtain:

$$
P_{s} T_{s} F(n, m)\left(D \frac{2 \pi^{2} n^{2}}{N^{2} P_{s}^{2}}+j \frac{2 \pi m}{M T_{s}}\right) \approx \sum_{k=0}^{K-1} c_{k} e^{-j \frac{2 \pi n p_{k}}{N P_{s}}} e^{-j \frac{2 \pi m t_{k}}{M T_{s}}} .
$$

Notice that on the right-hand side of (11) we have a linear combination of $K$ complex exponentials. Fixing, for example, $m=0$ we can again apply the annihilating filter method and compute the roots $e^{-j \frac{2 \pi p_{k}}{N T_{s}}}$ from which we obtain the positions $p_{k}$. Repeating the same steps for a fixed $n$, we can compute the time instants $t_{k}$. The coefficients $c_{k}$ can then be directly obtained from (11). We remark that the equation (11) is only an approximation of (10) since the DFT coefficients are aliased. The aliasing decreases as the time $t$ increases since $F\left(\Omega_{p}, \Omega_{t}\right)$ is becoming more and more bandlimited-like. This means that for an appropriate $t$, one can minimize the total effect of aliasing and noise, and optimize the estimation.

\subsection{Tracking}

Using the previous results different tracking algorithms can be imagined. For example, assume that a group of $K$ Diracs can appear at different times. If we sample the field over time fast enough, so that no more than one group of Diracs appear in the same sampling interval, then the exact reconstruction method can be derived from the results in 3.1. It is sufficient to compute the predicted field that is generated by the active sources at the previous state, i.e. $R g^{i-1}[n, t]$, and to subtract that from the current state $R g^{i}[n, t]$,

$R g_{i}[n, t]=R g^{i}[n, t]-R g^{i-1}[n, t]=\sum_{k=0}^{K-1} \frac{c_{k}}{\sqrt{4 \pi D\left(t-t_{i}\right)}} e^{-\frac{\left(n L-p_{k}\right)^{2}}{4 D\left(t-t_{i}\right)}}$

The resulting signal is a sum of $K$ Gaussians with the same variance $\sigma^{2}=2 D\left(t-t_{i}\right)$, and the relevant parameters are found by applying the algorithms in 3.1. If more than one Dirac with different time origin appear in the same sampling interval then the approximate results from 3.2 can be used.

\section{HEAT DIFFUSION AND ACOUSTIC TOMOGRAPHY}

\subsection{The estimation of Radon transform}

From a practical point of view, there is a close connection between the Radon transform projections of temperature distribution and acoustic tomography measurements. Namely, the typical time of flight measurements, provide the information about the corresponding Radon transform [6]. Assume that the acoustic transmitters and receivers are placed around a certain region of interest $D$, as shown on Fig. 1, and that we can measure the time of flight from the transmitter located at the position $q_{T}$ to the receiver located at the position $q_{R}=0$. Then,

$$
\tau=\int_{q_{R}}^{q_{T}} \frac{1}{c} d q
$$

where $c$ is the speed of sound. In dry air, the temperature $T$ can be inferred from the speed of sound through the relation

$$
c=\sqrt{R \gamma T},
$$

where $R$ is the gas constant and $\gamma=1.4$. Assume that at the instant $t_{0}=0$ the $K$ localized heat sources are active, and we want to estimate the temperature variation at time $t$. Applying the same model as before we have that $T(t)=T(0)+\Delta T$ and $\Delta T=g(x, y, t)$. Linearizing (12) and using (13), we have:

$\tau=\int_{q_{R}}^{q_{T}} \frac{1}{c_{0}} d q-\frac{1}{c_{0}^{2}} \int_{q_{R}}^{q_{T}} \Delta c d q=\int_{q_{R}}^{q_{T}} \frac{1}{c_{0}} d q-\frac{R \gamma}{2 c_{0}^{3}} \int_{q_{R}}^{q_{T}} \Delta T d q$,

where $c_{0}=\sqrt{R \gamma T(0)}$. If we define $\hat{R} g(x, y)$ as the Radon transform of $\hat{g}(x, y)=g(x, y)$ for $(x, y) \in D$ and $\hat{g}(x, y)=0$ outside, then

$$
\hat{R} g\left(p, \theta_{0}\right)=\frac{2 c_{0}^{3}}{R \gamma}\left(\int_{q_{R}}^{q_{T}} \frac{1}{c_{0}} d q-\tau\right) .
$$

We compute the Radon transform $\hat{R} g\left(p, \theta_{0}, t\right)$ in a similar way as before:

$$
\begin{aligned}
\hat{R} g\left(p, \theta_{0}, t\right) & =\int_{0}^{q_{T}} \sum_{k=0}^{K-1} \frac{c_{k}}{2 \pi \sigma^{2}} e^{-\frac{\left(p-p_{k}\right)^{2}+\left(q-q_{k}\right)^{2}}{4 D t}} d q \\
& =\sum_{k=0}^{K-1} \frac{c_{k}}{\sqrt{4 \pi D t}}\left(\operatorname{erf}\left(\frac{q_{k}}{\sqrt{4 D t}}\right)+\operatorname{erf}\left(\frac{q_{T}-q_{k}}{\sqrt{4 D t}}\right)\right) e^{-\frac{\left(p-p_{k}\right)^{2}}{4 D t}} .
\end{aligned}
$$




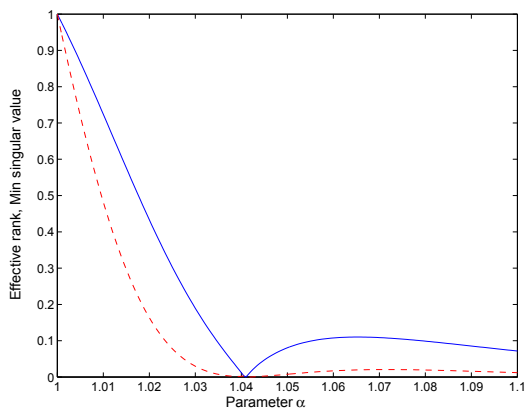

(a)

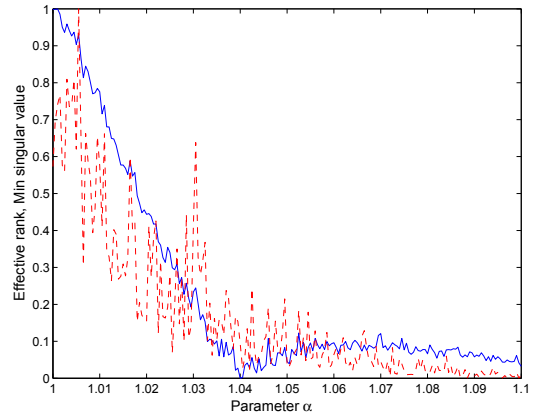

(b)

Fig. 2. Comparison of the time origin search algorithms: the first one searches for the $\alpha$ that minimizes the effective rank (plain) measure and the second one searches for the $\alpha$ that minimizes the smallest singular value (dashed). The optimal $\alpha$ is 1.04 . (a) Noiseless case. (b) Noisy case (SNR $=45 \mathrm{~dB}$ ).

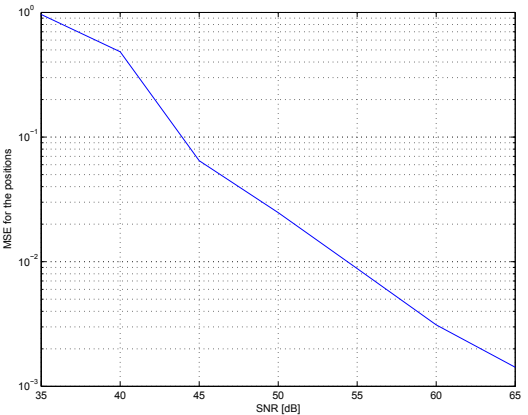

(a)

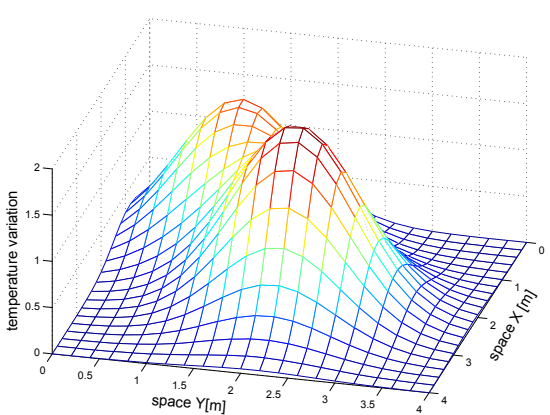

(b)

Fig. 3. (a) Relative MSE for the position estimation, (b) The reconstruction of two $2 \mathrm{D}$ Diracs. The samples of the Random transform are computed along the dashed lines.

Similarly,

$\hat{R} g\left(q, \theta_{0}+\frac{\pi}{2}, t\right)=\sum_{k=0}^{K-1} \frac{c_{k}}{\sqrt{4 \pi D t}}\left(\operatorname{erf}\left(\frac{p_{k}}{\sqrt{4 D t}}\right)+\operatorname{erf}\left(\frac{p_{T}-p_{k}}{\sqrt{4 D t}}\right)\right) e^{-\frac{\left(q-q_{k}\right)^{2}}{4 D t}}$

The parameters $\left\{c_{k}, p_{k}, q_{k}\right\}$ and $t_{0}$ can be found by applying the algorithms previously introduced in Sec. 3.1.

\subsection{Simulation results}

In the following, we present the simulation results. The setup consists of two local heat sources (2D Diracs). The parameters of the sources are randomly chosen in the corresponding intervals: $c_{k} \in$ $(0.5,10), x_{k}, y_{k} \in(0,4)$. All Diracs appear at the same time.

We first tested the time search algorithm. According to (9) we need to search for $\alpha$ that makes the matrix $\mathbf{S}$ rank deficient. In the case of the Effective Rank (ER) criterion, the minimum ER corresponds to the largest discrepancy in the eigenvalues distribution. Therefore, the optimal $\alpha$ is the one that contributes most to the clear differentiation between the signal and noise space. In Fig. 2, we plot the ER and the minimum eigenvalue of $\mathbf{S}$ with respect to $\alpha$. For comparison purpose, the matrix $\mathbf{S}$ is normalized and the results are scaled to the interval $[0,1]$. In the noiseless case, the two methods provide the correct answer $\alpha_{o p t}=1.04$ [Fig. 2(a)]. In the noisy scenario ( $\mathrm{SNR}=45 \mathrm{~dB}$ for the samples $R g[n]$ ), the ER clearly outperforms the singular value approach which basically provides no insight about the optimal solution [Fig. 2(b)].

Fig. 3(a) shows the performance of the position finding algorithm proposed in Sec. 3.1.1. This algorithm is known to be very unstable for the case of noisy data. Therefore, we use the successful variation of the original algorithm, that is developed in [7]. Good estimates are obtained by performing the oversampling in space with $\cdot N=5(2 K+1)$. The relative MSE represents the mean square of the relative error of the positions. The algorithm gives good results for SNR $>$ 40dB. Fig. 3(b) shows one reconstruction example.

\section{REFERENCES}

[1] M. Vetterli, P. Marziliano, and T. Blu, "Sampling signals with finite rate of innovation," IEEE Trans. on Signal Proc., vol. 50, no. 6, pp. 1417$1428,2002$.

[2] I. Maravic and M. Vetterli, "Exact Sampling Results for Some Classes of Parametric Non-Bandlimited 2-D Signals," IEEE Transactions on Signal Processing, vol. 52, no. 1, pp. 175-189, 2004.

[3] Dragotti P.L. and M. Vetterli, "Exact sampling results for signals with finite rate of innovation using stang-fix conditions and local kernels," in IEEE Conference on Acoustics, Speech and Signal Processing, 2005.

[4] I. Maravic and M. Vetterli, "A sampling theorem for the radon transform of finite complexity objects," in IEEE Conference on Acoustics, Speech and Signal Processing, 2002, vol. 2, pp. 1197-1200.

[5] O. Roy and M. Vetterli, "The effective rank," To appear in European Signal Processing Conference (EUSIPCO), 2007.

[6] I. Jovanovic, L. Sbaiz, and M. Vetterli, "Acoustic Tomography Method for Measuring Temperature and Wind Velocity," in IEEE Intern. Conf. on Acous., Speech, and Signal Proces., 2006.

[7] I. Maravic and M. Vetterli, "Sampling and Reconstruction of Signals with Finite Rate of Innovation in the Presence of Noise," IEEE Transactions on Signal Processing, vol. 53, no. 8, pp. 2788-2805, 2005. 